\title{
Prognostic Factors in Arthroplasty in the Rheumatoid Shoulder
}

\author{
Piet M. Rozing, MD, PhD • Jochem Nagels, MD • Maarten P. Rozing, MD
}

Received: 2 February 2010/Accepted: 10 June 2010/Published online: 14 July 2010

(C) The Author(s) 2010. This article is published with open access at Springerlink.com

\begin{abstract}
Total shoulder arthroplasty is commonly considered a good option for treatment of the rheumatoid shoulder. However, when the rotator cuff and glenoid bone stock are not preserved, the clinical outcome of arthroplasty in the rheumatoid patients remains unclear. Aim of the study is to explore the prognostic value of multiple preoperative and peroperative variables in total shoulder arthroplasty and shoulder hemiarthroplasty in rheumatoid patients. Clinical Hospital for Special Surgery Shoulder score was determined at different time points over a mean period of 6.5 years in 66 rheumatoid patients with total shoulder arthroplasty and 75 rheumatoid patients with shoulder hemiarthroplasty. Moreover, radiographic analysis was performed to assess the progression of humeral head migration and glenoid loosening. Advanced age and erosions or cysts at the $\mathrm{AC}$ joint at time of surgery were associated with a lower postoperative Clinical Hospital for Special Surgery Shoulder score. In total shoulder arthroplasty, status of the rotator cuff and its repair at surgery were
\end{abstract}

Each author certifies that he or she has no commercial associations (e.g., consultancies, stock ownership, equity interest, patent/licensing arrangements, etc.) that might pose a conflict of interest in connection with the submitted article.

Each author certifies that his or her institution has approved the reporting of these cases, that all investigations were conducted in conformity with ethical principles of research, and that informed consent for participating in the study was obtained.

Level of evidence of this study is: therapeutic study, level IV.

P. M. Rozing, MD, PhD .J. Nagels, MD

Department of Orthopaedic Surgery,

Leiden University Medical Center, PO Box 9600, 2300 RC, Leiden,

The Netherlands

M. P. Rozing, MD

Department of Gerontology and Geriatrics,

Leiden University Medical Center, PO Box 9600, 2300 RC, Leiden,

The Netherlands

P. M. Rozing, MD, $\mathrm{PhD}(\bowtie)$

PO Box 34, 2360 AA, Warmond-Teylingen,

The Netherlands

e-mail: P.M.Rozing@gmail.com predictive of postoperative improvement. Progression of proximal migration during the period after surgery was associated with a lower clinical score over time. However, in hemiarthroplasty, no relation was observed between the progression of proximal or medial migration during followup and the clinical score over time. Status of the AC joint and age at the time of surgery should be taken into account when considering shoulder arthroplasty in rheumatoid patients. Total shoulder arthroplasty in combination with good cuff repair yields comparable clinical results as total shoulder arthroplasty when the cuff is intact.

Keywords rheumatoid arthritis · rheumatoid shoulder. shoulder joint · prosthesis shoulder prosthesis .

shoulder arthroplasty- total shoulder prosthesis .

humeral head prosthesis $\cdot$ hemiarthroplasty.

glenoid component $\cdot$ loosening outcome measurement .

rotator cuff

\section{Introduction}

Total shoulder arthroplasty is considered a good option for treatment of the rheumatoid shoulder when the rotator cuff is intact and the glenoid bone stock is preserved. In rheumatoid shoulders with loss of glenoid bone stock, rupture of the rotator cuff, and/or rheumatoid destruction of the AC joint $[1,3,6]$, the clinical outcome of arthroplasty is often less predictable [13, 15].

Several factors have been implicated in affecting the prognosis following shoulder arthroplasty for patients with rheumatoid involvement. These include the degree of glenohumeral destruction, proximal and medial migration of the humeral head [5], preoperative status of the rotator cuff [16], successful repair of the cuff [9], involvement of the AC joint [3], age at the time of surgery [15], and sex. However, in most clinical follow-up studies, the mean postoperative result is based on only one postoperative measurement with a different follow-up period for most of the patients. However, the postoperative status of the patient often changes over time and as a result the postoperative score will gradually increase in some patients or decrease in others in the years after surgery. Therefore, at least two 
measurements are necessary in the years after surgery to assess the effect of prognostic factors on the trend of the score over time of an individual patient. More measurements will add to the accuracy by which a trend of the score over time of an individual patient can be estimated [9].

Therefore, in this prospective study, we analyzed the effect of various prognostic factors on the outcome of shoulder arthroplasty in patients with rheumatoid arthritis by using multiple functional measurements at different time points. Specifically, we studied the effect of the following preoperative and peroperative prognostic factors: $\mathrm{AC}$ joint destruction [3], age [15], glenoid bone loss [5], and status of the rotator cuff $[9,16]$ on the Hospital for Special Surgery clinical score (HSS) comparing the preoperative score to that obtained at 2 years of follow-up. Additionally, we studied the trend of the score over time in relation to the above-mentioned factors, to determine the change in HSS score over time.

\section{Patients and methods}

All rheumatoid patients in whom an arthroplasty of the shoulder was performed between 1985 and 2005 were prospectively studied. The group consisted of 156 primary shoulders in 124 patients. Thirty-two patients (42 shoulders) had died from unrelated causes in the years following surgery. Seven of these were not included in the follow-up study because they died within 2 years after surgery. Thirtyfive of these patients were regularly seen in the clinic for their follow-up and were included in the study. One patient with bilateral shoulder prosthesis refused to come for followup because she had no complaints. Three shoulders were considered as lost to follow-up because they could not be traced and of three shoulders the follow-up data were incomplete. Hence, 141 shoulders in 110 patients were included in this study (Table 1). There were 36 males and 74 females. The operation was performed in 68 right shoulders and 73 left shoulders, and in 64 cases, it concerned the dominant extremity. The average age was 60 years (range, 24 to 81 years). The follow-up period ranged from 2 to 20 years with a mean duration of follow-up of 6.5 years (SD 4 years). All patients were examined by the first author and scored clinically and radiographically before the operation, at 1-year and 2-year follow-up examinations and afterward for a 5and 10-year follow-up. The frequency of the scoring during follow-up was on average four times. In this way, the change of the score in the years after surgery could be evaluated for every individual patient. The Hospital for Special Surgery (HSS score) 100 point scoring system was used for clinical evaluation [7]. This 100-point scoring system allocates 30 points to pain, 25 points to power, 25 points to motion, and 20 points to function. A handheld goniometer was used to assess the range of motion and strength was measured with a hand held dynamometer and by manual muscle testing.

A standardized system of both performing and assessing the radiographs was used. All radiographs were assessed by two individuals (PMR, JN) and, in case of a discrepancy, were reevaluated until consensus was reached. It included a true antero-posterior view of the shoulder with the patient in
Table 1 Patient characteristics

\begin{tabular}{|c|c|c|}
\hline Type of arthroplasty & $\begin{array}{l}\text { Total shoulder } \\
\text { arthroplasty } \\
\text { Number of cases }\end{array}$ & $\begin{array}{l}\text { Hemiarthroplasty } \\
\text { Number of cases }\end{array}$ \\
\hline Number of operations & 66 & 75 \\
\hline Mean age $\pm \mathrm{SD}($ years $)$ & $56.9 \pm 12.85$ & $62.2 \pm 10.35$ \\
\hline Mean follow-up $\pm \mathrm{SD}$ (years) & $7.8 \pm 3.68$ & $5.5 \pm 3.96$ \\
\hline \multicolumn{3}{|l|}{ Radiol. grading } \\
\hline Larsen 3 & 4 & 4 \\
\hline Larsen 4 & 52 & 35 \\
\hline Larsen5 & 10 & 36 \\
\hline \multicolumn{3}{|l|}{$\mathrm{AC}$ joint destruction } \\
\hline Normal & 24 & 19 \\
\hline Cysts & 18 & 13 \\
\hline Erosions & 13 & 15 \\
\hline Acro-osteolysis & 9 & 19 \\
\hline Missing & 2 & 9 \\
\hline \multicolumn{3}{|c|}{ Med. migration (coracoid line) } \\
\hline None/mild $(\geq 0)$ & 24 & 14 \\
\hline Moderate $(-1 /-5)$ & 25 & 22 \\
\hline Severe $(<-5)$ & 17 & 37 \\
\hline Missing & & 2 \\
\hline \multicolumn{3}{|l|}{ Sup. migration (AH interval) } \\
\hline None/mild (>6 mm) & 19 & 16 \\
\hline Moderate $(4-6 \mathrm{~mm})$ & 27 & 18 \\
\hline Severe $(<3 \mathrm{~mm})$ & 19 & 37 \\
\hline Missing & 1 & 4 \\
\hline \multicolumn{3}{|l|}{ Pre-op. cuff } \\
\hline Intact & 31 & 17 \\
\hline Attenuated/intact & 10 & 10 \\
\hline Small tear & 10 & 11 \\
\hline Large tear & 15 & 34 \\
\hline Missing & & 3 \\
\hline \multicolumn{3}{|l|}{ Gender } \\
\hline Male & 18 & 28 \\
\hline Female & 48 & 47 \\
\hline \multicolumn{3}{|l|}{ Age } \\
\hline$\leq 50$ & 26 & 13 \\
\hline $51-60$ & 8 & 15 \\
\hline $61-70$ & 23 & 31 \\
\hline$>70$ & 9 & 16 \\
\hline
\end{tabular}

an upright position (with the back in $45^{\circ}$ to the roentgen film) and an axillary view. The AP view was taken with the humerus in external and internal rotation. In addition, an MRI or CT scan was obtained on most shoulders. The radiographic destruction of the shoulder joint was scored according to the criteria of Larsen et al. [4] (Larsen 0: normal shoulder; Larsen 1: soft tissue swelling and osteoporosis; Larsen 2: marginal erosions; Larsen 3: obvious narrowing of the joint space; Larsen 4: deep erosions through the subchondral plate and joint space reduced to a joint line; and Larsen 5: total disorganizations of joint and major bone destruction. Eight shoulders were graded as Larsen 3, 87 shoulders as Larsen 4, and 46 shoulders as Larsen 5. Superior migration of the humeral head was measured by the width of the acromio-humeral interval [5]. In five shoulders, the proximal migration could not be measured due to the inferior quality of the digitized radiographs. The change in proximal migration during follow-up was estimated by comparing the follow-up radiographs with the preoperative radiographs and measuring the width of the subacromial space and the position of 
the center of the humeral head opposite the glenoid. Three categories were distinguished: progression, no change, and improvement of proximal migration. The medialization or medial migration of the humeral head is caused by loss of the subchondral bone mass of the glenoid due to the rheumatoid process. The position of the joint surface can be measured relative to the projection of the basis of the coracoid process $[2,10]$. A vertical line is drawn tangential to the lateral margin of the coracoid process, and the distance between this line and the medial contour of the humeral head is measured. It gets a positive sign when the head is lateral to this line and a negative sign if the head is medial to this line. Also, a descriptive evaluation of glenoid bone loss was used in terms of the severity of the medial migration. There was no medial migration when there was no glenoid bone loss, mild medial migration when the joint line was lateral to the coracoid line, moderate medial migration when the joint line was $0-5 \mathrm{~mm}$ medial to the coracoid line, and severe medial migration when the joint line was more than $5 \mathrm{~mm}$ medial to this line. In 38 shoulders, the joint line was lateral to this coracoid line (no or mild medial migration); in 47 shoulders, the joint line was medialized between 0 and $5 \mathrm{~mm}$ (moderate medial migration), and in 54 shoulders, the medialization was more than $5 \mathrm{~mm}$ to this line (severe medial migration). In two shoulders, the medialization could not be measured accurately because the radiograph was not a true antero-posterior view. The $\mathrm{AC}$ joint was evaluated on standard radiographs of the shoulder for para-articular cysts, erosions, or acroosteolysis of the clavicle [1], which respectively represent progressive stages of rheumatoid destruction. No special views were made for evaluation of this joint. Three items were evaluated: para-articular cysts in 31 joints, erosions in 28 joints, acro-osteolysis of the clavicula in 28 joints, and a normal AC joint in 43 shoulders. On 11 shoulder radiographs, the visualization of the $\mathrm{AC}$ joint was insufficient for evaluation. The width of the joint space and the degree of subluxation of the AC-joint was not measured in this study.

All surgery was performed by the first author or by a resident under his supervision. The first and preferred option for treatment was total shoulder arthroplasty (TSA). Nevertheless, in 66 shoulders, a total shoulder arthroplasty was performed and, in 75 shoulders, a hemiarthroplasty. Hemiarthroplasty was performed when the bone loss of the glenoid did not allow secure fixation of the glenoid component or when a large irreparable cuff tear was present. Sometimes, the surgical plan was changed at the time of surgery in favor of a hemiarthroplasty when the surgical exposure of the glenoid was so insufficient risking compromise of insertion of the glenoid component. For replacement of the humeral head, two uncemented types of prosthesis were used. A biomodular humeral prosthesis was used in 83 shoulders (Biomodular Prosthesis, Biomet, Warsaw, IN) and an ESKA prosthesis with a short intramedullary stem in 58 shoulders (ESKA Implants $\mathrm{GmbH}$, Lübeck). There was no special indication for the use of one of them. Most of the components were used uncemented, and, in only eight shoulders (four Biomodular, four Eska), the humeral stem was cemented because the initial fixation at the time of surgery was considered insufficient for bony ingrowth. When a total shoulder prosthesis was inserted, a biomodular glenoid component was used, an uncemented metal backed version in 18 shoulders, and a cemented polyethylene keeled design in 48 shoulders. There was no special indication for use of an uncemented glenoid component.

The status of the rotator cuff was known preoperatively from the CT (arthro-) or MRI, and the cuff was also inspected at the time of surgery. In 48 shoulders, the cuff had a normal aspect; in 20 shoulders, the cuff was thin and attenuated but still intact, and, in 21 shoulders, it was combined with a small tear (diameter $<3 \mathrm{~cm}$ ). In 49 shoulders, a large tear was present (diameter $>3 \mathrm{~cm}$ ). In three shoulders, the cuff could not be reliably inspected. At the time of surgery, an attempt was made to repair the cuff tear. The quality of the rotator cuff after repair was subjectively graded as moderate if the cuff could not be closed completely, and a small defect remained or the thin and attenuated rotator cuff was closed under tension. The repair was graded as good if the cuff was closed without tension with strong tendon tissue, and intact if no tear in the cuff had to be closed. When no repair of the tear was performed, the quality of the cuff was graded as bad. In 49 shoulders with a large cuff tear, the repair was considered moderate in 14 shoulders and good in ten shoulders, and the tear was not repaired in 25 cases. In 21 shoulders with a small tear, the quality of the cuff after repair was graded as moderate in ten shoulders, good in four, and bad in seven.

The data of all patients were collected prospectively with MRDM software (Medical Research Data Management (www.clinicalresearch.nl) and converted into SPSS (SPSS, Chicago, Illinois) for data analysis. The difference between the preoperative and postoperative HSS score and its sub-items was tested using the Student's $t$ test. Correlation analysis was used for measuring the degree of association between the preoperative and postoperative clinical score with the Spearman's rank correlation coefficient. The relationship between improvement of the clinical score after operation and prognostic factors was analyzed with the one-way analysis of variance with the preoperative score as covariate for categorical data and linear regression for numerical data. The Pearson chi-square test for independence was used for the relationship of the preoperative, peroperative, and postoperative categorical data to one another. Comparison of the clinical score after TSA and hemiarthroplasty (HHR) in patients with bilateral shoulder prosthesis was done with the paired $t$ test. The change of the clinical score during follow-up in relation with the prognostic factors like type of arthroplasty, superior and medial migration, cuff status, radiographic destruction, sex, and age were analyzed by means of a generalized linear mixed model with the presurgery status as a covariate. In this way, the trend of the score or the change of the score with the passage of time was studied within the different groups and subgroups of the variables as well as between the different groups. Level of significance was set at 0.05 for all analyses. 
Table 2 Comparison of the average HSS score after Total Shoulder Arthroplasty and Humeral Head Replacement

\begin{tabular}{|c|c|c|c|c|c|c|}
\hline \multirow{2}{*}{$\begin{array}{l}\text { Arthroplasty } \\
\text { type }\end{array}$} & \multirow{2}{*}{$\begin{array}{l}\text { HSS clinical } \\
\text { score }\end{array}$} & \multicolumn{5}{|l|}{ HSS score } \\
\hline & & Preoperative & 2 years & 5 years & 10 years & $p$ for trend \\
\hline \multirow[t]{5}{*}{ HHR $(n=75)$} & Total $(0-100)$ & 42.3 & 69.5 & 71.8 & 73.0 & 0.534 \\
\hline & Pain $(0-30)$ & 10.1 & 25.6 & 25.1 & 26.9 & 0.259 \\
\hline & Motion $(0-25)$ & 9.1 & 12.6 & $14.1 *$ & $13.3 * *$ & 0.412 \\
\hline & Function $(0-20)$ & 7.3 & 13.6 & 13.5 & 13.3 & 0.764 \\
\hline & Strength $(0-25)$ & 11.7 & 12.6 & 13.6 & 13.0 & 0.202 \\
\hline \multirow[t]{5}{*}{ TSA $(n=66)$} & Total $(0-100)$ & 40.9 & 70.0 & 69.2 & 66.0 & 0.045 \\
\hline & Pain $(0-30)$ & 9.0 & 25.6 & 24.3 & 23.8 & 0.068 \\
\hline & Motion $(0-25)$ & 8.9 & 11.5 & $12.1 *$ & $11.1 * *$ & 0.555 \\
\hline & Function $(0-20)$ & 7.4 & 13.6 & 14.3 & 13.1 & 0.041 \\
\hline & Strength $(0-25)$ & 12.4 & 13.0 & 12.9 & 12.3 & 0.089 \\
\hline
\end{tabular}

Data are presented as mean HSS score with standard error of the mean. Difference in motion between TSA and HHR, ${ }^{*} p=0.005 * * p=0.013$

\section{Results}

There was no significant difference in the pre-op HSS score and the sub-items, pain, motion, function, and strength comparing HHR and TSA in the whole group of patients (Table 2). The HSS score and its sub-items pain, motion, function and strength, and the change of the preoperative scores were significantly improved at the 2 years follow-up compared with the preoperative scores (Student's $t$-test: $p<$ $0.001)$. After this initial improvement, there was a gradual decrease of the HSS score $(p=0.045)$ and the function score $(p=0.041)$ over time in patients with TSA. In shoulders with HHR, there was no significant change of the score over time (Table 2). In the group of patients with bilateral shoulder prosthesis, there were 11 patients with a TSA in one shoulder and a HHR in the other shoulder. In these 11 patients, no significant difference was found between both shoulders regarding the HSS score and the score of the subitems both preoperatively and at the 2 years follow-up (paired $t$-test HSS score: preoperative, $p=0.751$; postoperative, $p=0.772$ ). The importance of the prognostic values was studied in shoulders with TSA and HHR. In TSA, the improvement of the score was positively correlated with the status of the rotator cuff after insertion of the prosthesis $(p=$ $0.006)$ as well as younger age at the time of surgery $(p<$ 0.001). In HHR, the postoperative improvement varied significantly with respect to the preoperative medial migration $(p=0.019)$ and the status of the AC joint $(p=0.022)$.

The preoperative status of the $\mathrm{AC}$ joint was significantly related to the clinical outcome after arthroplasty $(p=0.034)$. In patients with HHR, the presence of erosions or cysts in the $\mathrm{AC}$ joint on the preoperative radiographs was associated with less improvement of the HSS score in comparison with those with an intact $\mathrm{AC}$ joint $(p=0.005)$ and was associated with a lower HSS score during the whole follow-up ( $p=$ 0.004). In patients with TSA, the improvement of the score at the 2-year follow-up did not vary with the status of the $\mathrm{AC}$ joint at the time of surgery $(p=0.509)$, but there was a gradual decrease of the score with the passage of time in shoulders with degenerative changes in the $\mathrm{AC}$ joint at the time of surgery $(p=0.030)$.
Age at the time of surgery influenced the improvement of the score after surgery significantly in TSA $(p<0.001)$ and HHR $(p=0.036$; Fig. 1$)$. In patients with TSA and younger than 50 years, the improvement of the HSS score was $34.6(\mathrm{SD}, 13.60)$ at the 2 years follow-up and in patients older than 70 years 22.9 (SD, 18.03). Although the improvement of the score after surgery differed with age at the time of surgery, age was not a factor in deterioration of the score with the passage of time after TSA or HHR.

Outcome of TSA was related to the integrity of the rotator cuff. Patients with TSA showed significantly less improvement of the score at the 2 years follow-up in shoulders with a moderate repair of the cuff than in shoulders with a good repair or an intact cuff $(p=0.006)$.

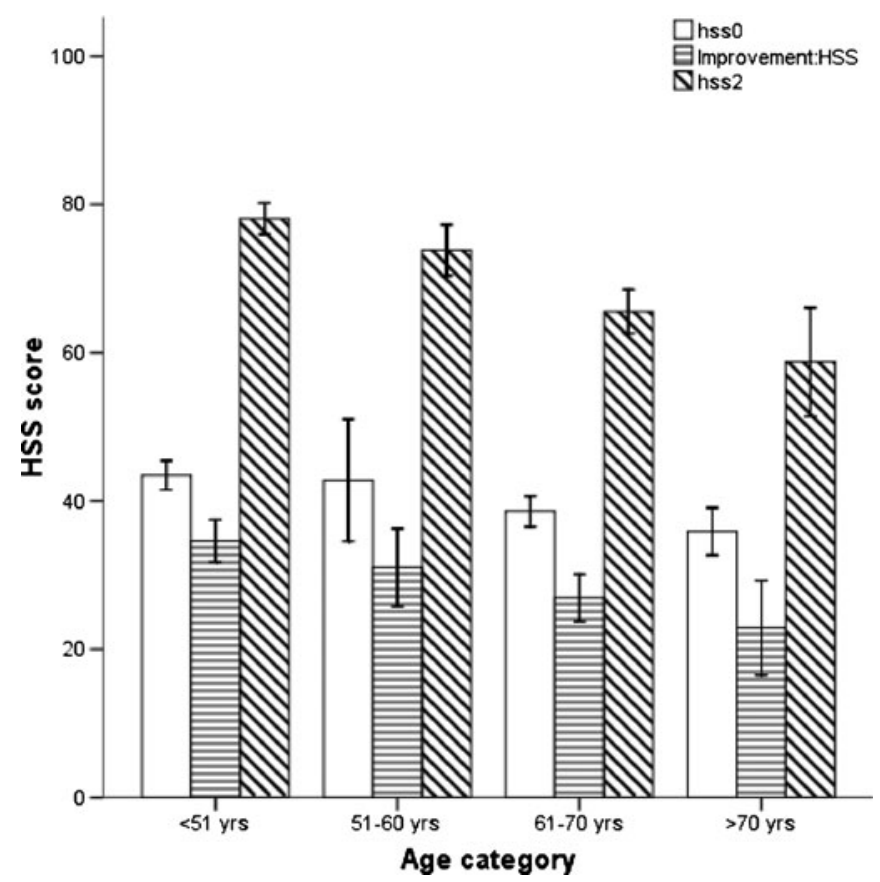

Fig. 1. The relationship between age and clinical outcome after shoulder replacement is shown. (White bar: preoperative HSS score; transverse striated bar: postoperative improvement; oblique striated bar: HSS score at 2 years follow-up). Data are presented as mean HSS score with standard error of the mean 
Table 3 Cuff repair versus pre- and postoperative HSS score for TSA and HHR

\begin{tabular}{|c|c|c|c|c|c|c|}
\hline \multirow{2}{*}{ Arthroplasty type } & \multirow[t]{2}{*}{ Cuff repair } & \multicolumn{5}{|c|}{ HSS score \pm standard deviation } \\
\hline & & Preoperative & 2 years & 5 years & 10 years & $p$ for trend \\
\hline HHR $(n=32)$ & $\mathrm{Bad}$ & $43.5 \pm 14.49$ & $72.3 \pm 14.56^{*}$ & $76.0 \pm 11.64$ & $70.5 \pm 13.0$ & 0.753 \\
\hline $\operatorname{HHR}(n=12)$ & Moderate & $42.8 \pm 12.55$ & $70.3 \pm 13.73 *$ & $68.1 \pm 19.28$ & $71.3 \pm 20.1$ & 0.647 \\
\hline $\operatorname{HHR}(n=2)$ & Good & $57.5 \pm 6.36$ & $84.5 \pm 3.53 *$ & 78.5 & - & 0.607 \\
\hline HHR $(n=26)$ & Intact & $39.9 \pm 12.96$ & $66.7 \pm 13.25^{*}$ & $69.6 \pm 11.17$ & $78.2 \pm 9.85$ & 0.520 \\
\hline TSA $(n=0)$ & Bad & - & - & - & - & - \\
\hline TSA $(n=13)$ & Moderate & $38.4 \pm 14.83$ & $57.0 \pm 14.79 * *$ & $54.5 \pm 19.3$ & $56.2 \pm 33.39$ & 0.859 \\
\hline TSA $(n=12)$ & Good & $40.0 \pm 9.63$ & $73.8 \pm 9.33 * *$ & $76.2 \pm 9.17$ & $59.5 \pm 14.50$ & 0.003 \\
\hline TSA $(n=41)$ & Intact & $41.9 \pm 9.47$ & $73.6 \pm 13.96 * *$ & $72.0 \pm 14.44$ & $70.1 \pm 14.81$ & 0.122 \\
\hline
\end{tabular}

Relationship between cuff status after repair and improvement of HSS score in patients with TSA $(* * p=0.006)$ and patients with HHR $(* p=$ 0.704). Data are presented as mean HSS score with standard error of the mean. Significant decrease of the score over time for patients with TSA and a good repair of the rotator cuff $(p=0.003)$. Missing three shoulders with HHR

Shoulders with a good repair had a good improvement of the clinical score at the 2 years follow-up comparable with shoulders with an intact cuff (Table 3). After this initial improvement, the HSS score decreased over time in shoulders with a good repair with an average of 1.3 point/ year $(p=0.003)$. In patients with an intact cuff, the decrease of the score (on average 0.4 point/year) was not significant ( $p=$ 0.122). In patients with HHR, the improvement of the score did not vary with the quality of the repair $(p=0.704)$, and there was no significant change of the score over time in the different groups during follow-up (Table 3).

The intention at the time of surgery was always to replace the glenoid unless the repair of the cuff or the glenoid bone stock was considered insufficient for a reliable functioning of a TSA. Shoulders with a moderate repair of the cuff and TSA formed a positive selected group of patients in whom the authors expected a better outcome than in patients with HHR. Nevertheless, comparing the two groups with reasonable repair and with or without a glenoid component, patients with HHR had a higher HSS score during the whole follow-up ( $p=0.031$; Table 3 ).

In patients with TSA or HHR and an intact cuff at surgery, the degree of preoperative proximal migration was not significantly related with the improvement of the overall clinical HSS score or the height of the score at the 2 years follow-up. Regarding the sub-items of the score, in patients with TSA, the improvement of motion varied by the degree of preoperative proximal migration $(p=0.037)$, and shoulders with preoperative proximal migration had less flexion $(p=$ $0.009)$ and less abduction $(p=0.021)$ at the 2 years followup. If the acromio-humeral distance was less than $6 \mathrm{~mm}$, flexion was $81^{\circ} \pm 32^{\circ}$ (mean \pm standard deviation), and abduction $65^{\circ} \pm 20^{\circ}$, and if this distance was more than $6 \mathrm{~mm}$, flexion was $100^{\circ} \pm 36^{\circ}$ and abduction $86^{\circ} \pm 31^{\circ}$. In shoulders with HHR, the improvement of function was significantly better in shoulders with less preoperative proximal migration $(p=0.021)$.

Proximal migration at follow-up was significantly related with the degree of proximal migration preoperatively $(p<0.001)$ and older age at the time of surgery $(p<$ $0.001)$ ). In 39 shoulders, the proximal migration of the humeral head had progressed in the years after surgery, and in 38 shoulders, the proximal migration had improved in comparison with the preoperative proximal migration. Improvement occurred more frequently after a good repair of the cuff than after a moderate repair $(p=0.027)$. In patients with TSA, progression of proximal migration was accompanied with a decrease of the clinical score over time $(p=0.047)$, and the score was significantly lower in comparison with the score in patients with an improvement of proximal migration ( $p=0.034$; Fig. 2). In patients with HHR, the clinical score was not affected by an improvement or worsening of the proximal migration.

In patients with TSA, the improvement of the score did not vary with the degree of the preoperative medial migration $(p=0.564)$, and there was no significant difference in the trend of the scores during follow-up between shoulders with the glenoid surface lateral to the coracoid line (no or mild medial migration) and shoulders with bone loss medial to coracoid line [moderate or severe medial migration $(p=0.367)]$. In patients with HHR, the clinical score after surgery varied with the degree of medial migration by glenoid bone loss . The HSS score was higher and improved more after surgery in shoulders with moderate or severe medial migration than in shoulders with no or mild medial migration $(p=0.020)$. This finding could not be explained by a difference in distribution of frequency of age, cuff destruction, or cuff repair. The average HSS score during follow-up remained significantly lower in patients with preoperatively no or only mild glenoid bone loss $(p=0.041)$.

The medial migration measured at follow-up was compared with the preoperative migration in shoulders with a hemiarthroplasty. There was an increase of medialization at follow-up in 28 of 72 shoulders with a hemiarthroplasty (three missing), and the average progression measured in this group was $4.8 \mathrm{~mm}$ (SD, $3.4 \mathrm{~mm}$.). In 14 shoulders, it progressed to severe. A progression of the medial migration did not result in a decrease of the HSS score (Fig. 3) or the score of the sub-items pain, motion, function, or strength over time, and there was no significant difference in the trend of the clinical score and the score of sub-items between the shoulders that migrated medially and those shoulders that did not in the years after surgery HSS, $p=$ 


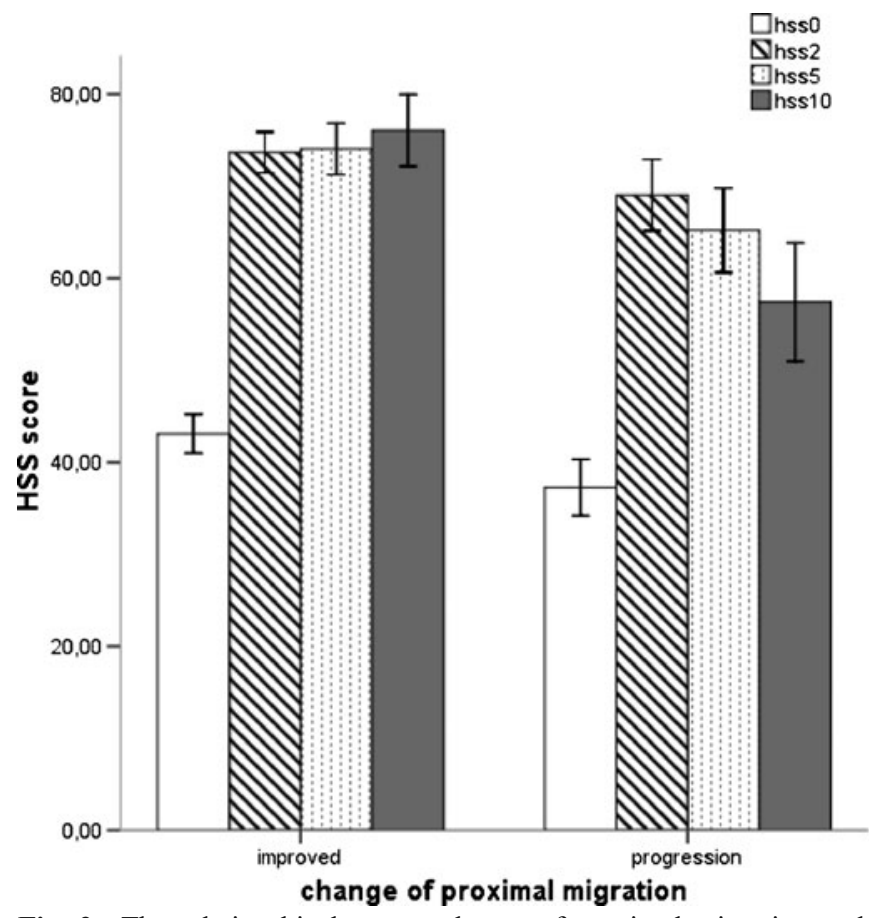

Fig. 2. The relationship between change of proximal migration and HSS score, preoperatively and at 2, 5, and 10 years follow-up in shoulders with TSA is demonstrated. Data are presented as mean HSS scores with standard error of the mean. Number of shoulders for different postoperative intervals are: for 0 years, 27 improved/16 progression; 2 years, 23/17; 5 years, 23/16; and 10 years, 13/11

0.90; pain, $p=0.98$; motion, $p=0.19$; function, $p=0.32$; and strength, $p=0.86$.

In the group with an uncemented glenoid component ( $n=18$ of 66 TSA's) with a follow-up of 7.9 years (SD, 3.97), there were no complications regarding the glenoid and no radiographic signs of loosening except in one patient in which there was a non-progressive resorption of bone under the metal backing. In patients with a cemented glenoid component $(n=48)$ with a follow-up of 7.1 years (SD 3.24 years), 17 of the cemented glenoid components had no radiolucency at the cement bone interface, and ten components a partial radiolucency. In 21 shoulders, the glenoid component was considered loose based upon a complete radiolucency, combined with a shift of the component in a superior tilt in 13 shoulders and additionally with major bone loss in nine shoulders. The presence of partial or complete radiolucency and loosening in cemented glenoid components was significantly related with the preoperative status of the rotator cuff. In shoulders with a preoperatively attenuated cuff (degenerated, small tear, or large tear), there was more often a loosening or radiolucency at follow-up $(p=0.002)$. This negative effect was not undone by a good repair of the rotator cuff and even in shoulders with a good repair of the cuff there was more often radiolucency or loosening at follow-up than in shoulders with an intact cuff $(p=0.004)$. The importance of the status of the rotator cuff is also expressed by the increased occurrence of glenoid loosening in shoulders with postoperatively a proximal migration $(p<0.001)$. The presence of medial migration of the humeral head preoper- atively caused by bone loss of the glenoid was significantly related with radiolucency and loosening of the glenoid component at follow-up $(p=0.042)$. Loosening of a cemented glenoid component occurred in shoulders with the joint line preoperatively lateral to the coracoid line in four out of 17 shoulders (relative risk $=0.31$ ) and in shoulders with the joint line medial to the coracoid line in 17 of 31 shoulders (relative risk=1.21). Of the 18 shoulders with an uncemented glenoid component, 11 shoulders had preoperatively a migration medial to the coracoid line but no loosening occurred. Patients with loosening of the glenoid component developed a lower HSS score over time $(p=0.022)$ and had a loss of function $(p=$ 0.001 ) in comparison with patients with a well-fixed glenoid.

In 11 shoulders, a revision operation was performed. Three revisions of a symptomatic loose glenoid component, one painful medial migration, one painful proximal migration, three cases with an acute rupture of the cuff, one release of an internal rotation contracture, and two cases with a painful $\mathrm{AC}$ joint.

\section{Discussion}

In this study, we aimed to investigate the prognostic value of various parameters in the clinical course after shoulder arthroplasty in rheumatoid patients. By using multiple postoperative functional measurements at different time points, we found that age at the time of surgery, the condition of the $\mathrm{AC}$ joint, the status of the rotator cuff, and

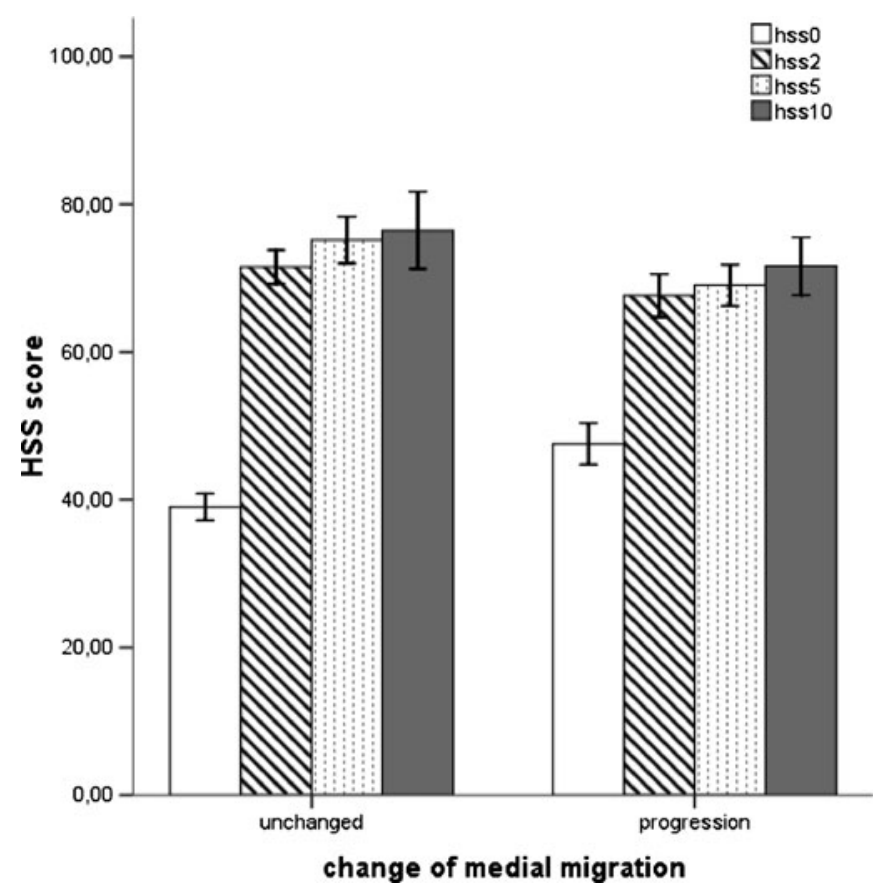

Fig. 3. The relationship between change of medial migration and HSS score preoperatively and at 2.5 and 10 years follow-up in shoulders with HHR. Data are presented as mean HSS scores with standard errors of the mean. The numbers of shoulders for different postoperative intervals are: for 0 years, 72 shoulders (44 unchanged/ 28 progression); 2 years, 64 (39/25); 5 years, 38 (19/19); 10 years, $16(7 / 9)$ 
the presence of a good glenoid bone stock were important predictors for the outcome during follow-up. Moreover, in patients with hemiarthroplasty, no deterioration of the clinical score over time was observed.

A possible limitation of this study is the low number of patients in certain subgroups and the non-randomization of the type of glenoid component used. Because this study was performed in a referral center for rheumatoid patients, more severely affected patients were included.

The age at the time of surgery is important for the improvement of the score after shoulder replacement, but it does not influence the trend of the score in the years after surgery.

The pathology of the AC joint in the rheumatoid shoulder has been reported by several authors $[1,3,6]$, but not in combination with a replacement arthroplasty of the shoulder. In these earlier studies, destruction of the AC joint and the glenohumeral joint do not follow the same course neither in time nor in severity of destruction. In our study, presence of radiological changes in the AC joint was an important prognostic factor. There might have been an indication to combine shoulder replacement surgery with a resection arthroplasty of the AC joint. More clinical studies are needed to confirm this statement.

In patients with HHR, the outcome of the HSS clinical score during follow-up was affected by the degree of preoperative medial migration by glenoid bone loss. In patients with HHR, the improvement of the score after surgery was less in shoulders with preoperatively none or mild medial migration. The reason for this finding is not clear. Progression of the medial migration during follow-up in patients with HHR did not result in a worsening of the clinical score in the years after surgery, contrary to findings in other reported series $[13,15]$. In our study, only one patient underwent a revision for this reason. Loss of glenoid bone stock might be a disadvantage in TSA. If the preoperative medial migration is beyond the base of the coracoids process, the long-term fixation of the glenoid component might be compromised as seen in this study. We also found that the presence of proximal migration at follow-up is associated with glenoid loosening $[8,12]$. The rate of loosening of the cemented glenoid component of $44 \%$ in our study is similar as reported in the literature $[7,8$, $14,15]$. Loosening had a significant influence on the outcome and caused a lower HHS score and a loss of function. This is contrary to previous findings suggesting that loosening did not influence pain relief, motion, function, or abduction strength [11].

Rheumatic involvement leading to disuse atrophy of the intact rotator cuff results in an anterior superior sublimation of the humeral head as is seen frequently in the rheumatoid shoulder [16]. In one shoulder study [15], it was found that the risk of proximal migration after surgery increased with older age and more severe migration preoperatively and was stated that proximal migration could occur irrespective of the status of the rotator cuff, which findings were confirmed in our series. Some authors [12] reported proximal migration after arthroplasty as the most frequent complication. In the present study, proximal migration was present preoper- atively in $75 \%$ of the shoulders in a degree of moderate or severe (AH interval $\leq 6 \mathrm{~mm}$ ), and there was postoperatively an improvement of the proximal migration compared with the preoperative status in $28 \%$ and a progression in $33 \%$ of the shoulders. An improvement of proximal migration was related with a good cuff repair. A progression of proximal migration resulted in a lower clinical score and a falling score during follow-up in patients with TSA. In agreement with earlier studies, progression of proximal migration did not affect the outcome in HHR [11].

In summary, the status of the rotator cuff is predictive of the outcome of TSA. At the time of surgery, if the quality of the rotator cuff after repair is assessed as moderate, HHR should be considered the procedure of choice. Glenoid bone loss increased the risk of glenoid loosening of cemented glenoid prosthesis in TSA, and in those cases, HHR is a good option for treatment. In HHR, no relation was observed between the progression of proximal or medial migration during follow-up and the clinical score over time. Age was a predictive factor for the improvement of the HSS score, and the status of the AC joint was related with the trend of the score over time.

Acknowledgements We thank R. Brand, $\mathrm{PhD}$, for his statistical advice.

Open Access This article is distributed under the terms of the Creative Commons Attribution Noncommercial License which permits any noncommercial use, distribution, and reproduction in any medium, provided the original author(s) and source are credited.

\section{References}

1. Dijkstra J, Dijkstra PF, vd KW. Rheumatoid arthritis of the shoulder. Description and standard radiographs. Rofo 1985;142 (2):179-85.

2. Figgie III HE, Inglis AE, Goldberg VM, Ranawat CS, Figgie MP, Wile JM. An analysis of factors affecting the long-term results of total shoulder arthroplasty in inflammatory arthritis. Journal of Arthroplasty 1988;3(2):123-130.

3. Kelly I.G. The source of shoulder pain in rheumatoid arthritis: Usefulness of local anesthetic injections. J Shoulder Elbow Surg 1994;3(2):62-65.

4. Larsen A, Dale K, Eek M. Radiographic evaluation of rheumatoid arthritis and related conditions by standard reference films. Acta Radiol Diagn (Stockh) 1977;18(4):481-491.

5. Lehtinen JT, Belt EA, Kauppi MJ, Kaarela K, Kuusela PP, Kautiainen HJ, et al. Bone destruction, upward migration, and medialisation of rheumatoid shoulder: a 15 year follow up study. Ann Rheum Dis 2001;60(4):322-326.

6. Lehtinen JT, Kaarela K, Belt EA, Kautiainen HJ, Kauppi MJ, Lehto MU. Relation of glenohumeral and acromioclavicular joint destruction in rheumatoid shoulder. A 15 year follow up study. Ann Rheum Dis 2000;59(2):158-160.

7. McCoy SR, Warren RF, Bade HA, III, Ranawat CS, Inglis AE. Total shoulder arthroplasty in rheumatoid arthritis. J Arthroplasty 1989;4(2):105-113.

8. Nagels J, Valstar ER, Stokdijk M, Rozing PM. Patterns of loosening of the glenoid component. J Bone Joint Surg Br 2002;84(1):83-87.

9. Rozing PM, Brand R. Rotator cuff repair during shoulder arthroplasty in rheumatoid arthritis. Journal of Arthroplasty 1998;13(3):311-319. 
10. Rozing PM, Obermann WR. Osteometry of the glenohumeral joint. J Shoulder Elbow Surg 1999;8(5):438-442.

11. Sneppen O, Fruensgaard S, Johannsen HV, Olsen BS, Sojbjerg $\mathrm{JO}$, Andersen NH. Total shoulder replacement in rheumatoid arthritis: proximal migration and loosening. J Shoulder Elbow Surg 1996;5(1):47-52.

12. Sojbjerg JO, Frich LH, Johannsen HV, Sneppen O. Late results of total shoulder replacement in patients with rheumatoid arthritis. Clin Orthop Relat Res 1999;(366):39-45.

13. Sperling JW, Cofield RH, Schleck CD, Harmsen WS. Total shoulder arthroplasty versus hemiarthroplasty for rheumatoid arthritis of the shoulder: Results of 303 consecutive cases. Journal of Shoulder and Elbow Surgery 2007;16(6):683-690.

14. Torchia ME, Cofield RH, Settergren CR. Total shoulder arthroplasty with the Neer prosthesis: Long-term results. Journal of Shoulder and Elbow Surgery 1997;6(6):495-505.

15. Trail IA, Nuttall D. The results of shoulder arthroplasty in patients with rheumatoid arthritis. Journal of Bone and Joint SurgerySeries B 2002;84(8):1121-1125.

16. van de Sande MA, de Groot JH, Rozing PM. Clinical implications of rotator cuff degeneration in the rheumatic shoulder. Arthritis Rheum 2008;59(3):317-324. 